\title{
Massive increase in serum creatine kinase during olanzapine and quetiapine treatment, not during treatment with clozapine
}

Received: 3 February 2000 / Accepted: 4 April 2000 / Published online: 19 May 2000

(C) Springer-Verlag 2000

Sir. Within the last years, there have been several publications describing creatine kinase (CPK) increases associated with neuroleptic treatment in the absence of neuroleptic malignant syndrome (NMS). Meltzer et al. (1996) reported a marked increase in CPK levels in approximately $10 \%$ of patients treated with clozapine, risperidone, melperone, olanzapine, haloperidol, or loxapine. In three cases, treatment with olanzapine was associated with an increase in CPK without NMS. There have also been reports associating olanzapine with NMS (Johnson and Bruxner 1998; Emborg and Kongevej 1999; Hickey et al 1999). In this letter, we describe a case in which there was a massive CPK increase during olanzapine treatment without symptoms of NMS, which disappeared during treatment with clozapine and returned after starting quetiapine.

\section{Case report}

Mr. A, a 19-year-old African-Caribbean man was admitted to our hospital because of undifferentiated schizophrenia according to DSM-IV (Diagnostic and Statistical Manual of Mental Disorders). $\mathrm{He}$ had been treated in an other hospital with risperidone (6 mg/day) and, on admission, he used olanzapine (20 mg/day). Both medicines he used for more than 6 weeks without response. On physical examination at admission, there were no particular findings. Routine laboratory tests exhibited a CPK of $6840 \mathrm{U} / 1$ (day 3 of hospitalization), which is 58 times the upper limit of normal. Further tests showed a relatively low amount of neutrophils $29.3 \%$ (normal range 40-72\%) - a serum glutamic oxaloacetic transaminase (SGOT) level of 112 , a serum glutamic pyruvic transaminase (SGPT) level of 43, and a lactate dehydrogenase (LDH) level of 231. There were no clinical manifestations of NMS: he had a normal temperature, there were no physical complaints, and no motor symptoms. There were no other indications for the most common reasons of CPK elevation: although the patient was severely psychotic he was not agitated or anxious, trauma was absent, there was no period of excessive physical activity, and he had not received any physical restraints or intramuscular

E. Boot $\cdot$ L. de Haan (

Academic Medical Center, University of Amsterdam,

Department of Psychiatry, Postbox 22700, 1100 DE Amsterdam,

The Netherlands injections. We lowered the dose to $10 \mathrm{mg} /$ day on day 19. The CPK first fluctuated between $500 \mathrm{U} / 1$ and $2000 \mathrm{U} / 1$. When the dose was lowered to $10 \mathrm{mg} / \mathrm{day}$, the lowest level measured was 679. Assessment showed $100 \%$ activity of the skeletal muscle (MM) type. Urine myoglobin was negative. Since the psychotic symptoms did not diminish with treatment of risperidone and olanzapine, we started clozapine treatment on day 29 , notwithstanding the low amount of neutrophils. The CPK decreased to about 300 and remained at that level.

On day 120 , we had to stop treatment of clozapine because the patient developed granulocytopenia. We started with quetiapine and the CPK mounted again to $3942 \mathrm{U} / 1$, and decreased after 4 days to $389 \mathrm{U} / 1$. It could be that the mechanism through which the CPK increases is not comparable for olanzapine, quetiapine, and clozapine. However, Meltzer et al. (1996) described three cases in which the increases were self limiting, despite continuing treatment. The CPK level during treatment with clozapine may be the baseline level for this man. Heightened upper limits of normal CPK were reported in African-American males (Black et al. 1986; Meltzer et al. 1996). Nevertheless, the factors related to antipsychotic medication that influence the CPK level are not known.

\section{Discussion}

Meltzer et. al (1996) suggested that the increase in CPK activity may reflect the ability of atypical antipsychotic agents to increase intermittently cell membrane permeability, especially in skeletal muscle, in vulnerable subjects. Keshavan et al. (1994) suggested that the isolated CPK elevations are likely to be related to muscle damage not severe enough to cause frank rhabdomyolysis, while Fogel and Goldberg (1985) suggested that NMS is the extreme end of a spectrum with many mild forms.

Since Meltzer et al. (1996) observed no clinical consequences of this elevation of CPK activity, they proposed that routine monitoring of CPK activity during treatment with the aforementioned antipsychotic drugs is probably not necessary. However, the long-term course and consequences of this side effect remain unknown. We suggest a periodical evaluation of clinical symptoms and CPK activity over a longer period of time in patients with a markedly elevated CPK activity who continue that 
medication to answer the question about the long-term course.

\section{References}

Black HR, Quallich H, Gareleck CB (1986) Racial differences in serum creatine kinase levels. Am J Med 81:479-487

Emborg C, Kongevej B (1999) Neuroleptic malignant syndrome after treatment with olanzapine. Ugeskr Laeger 161:14241425
Fogel BS, Goldberg RJ (1985) Neuroleptic malignant syndrome. N Engl J Med 313:1292

Hickey C, Stewart C, Lippmann S (1999) Olanzapine and NMS. Psychiatr Serv 50:836-837

Johnson V, Bruxner G (1998) Neuroleptic malignant syndrome associated with olanzapine. Aust NZJ Psychiatry 32:884-886

Keshavan MS, Stecker J, Kambhampati RK (1994) Creatine kinase elevations with clozapine. Br J Psychiatry 164:118120

Meltzer HY, Cola PA, Parsa M (1996) Marked elevations of serum creatine kinase activity associated with antipsychotic drug treatment. Neuropsychopharmacology 15:395-405 